\title{
Immunoregulatory therapy strategies that target cytokine storms in patients with COVID-19 (Review)
}

\author{
XIANYAO WANG ${ }^{1-3}$, ZHIXU HE $^{2,4}$ and XING ZHAO ${ }^{1-3}$ \\ ${ }^{1}$ Center for Tissue Engineering and Stem Cell Research, Guizhou Medical University; ${ }^{2}$ National Joint Local Engineering \\ Laboratory for Cell Engineering and Biomedicine Technique, Guizhou Province Key Laboratory of Regenerative Medicine, \\ Key Laboratory of Adult Stem Cell Translational Research, Chinese Academy of Medical Sciences, Guiyang, \\ Guizhou 550004; ${ }^{3}$ Department of Immunology, Guizhou Medical University, Guiyang, Guizhou 550025; \\ ${ }^{4}$ Department of Pediatrics, Affiliated Hospital of Zunyi Medical University, Zunyi, Guizhou 563000, P.R. China
}

Received September 18, 2020; Accepted December 1, 2020

DOI: $10.3892 /$ etm.2021.9750

\begin{abstract}
A cytokine storm is an uncontrolled, excessive immune response that contributes to the pathogenesis of coronavirus disease 2019 (COVID-19). Viral infections lead to the loss of negative feedback in immune regulation and an abnormal elevation of the levels of multiple cytokines. In COVID-19, this causes diffuse damage to alveolar functions and may culminate in multiple organ dysfunction. Immunoregulatory therapies target the cytokine storms induced by severe acute respiratory syndrome coronavirus 2 (SARS-CoV-2), the virus that causes COVID-19, and include monoclonal antibodies, recombinant granulocyte-macrophage colony stimulating factor, interferon, mesenchymal stem cell-based therapy, thymosin, immunoglobulins and blood purification therapies. These approaches may be effective in the alleviation of COVID-19 symptoms. In this review, cytokine storms caused by SARS-CoV-2 infections are evaluated and discussed, and advances in immunoregulatory therapy strategies for patients with COVID-19 are reviewed.
\end{abstract}

\section{Contents}

1. Introduction

Correspondence to: Professor Xing Zhao, Center for Tissue Engineering and Stem Cell Research, Guizhou Medical University, 9 Beijing Road, Guiyang, Guizhou 550004, P.R. China

E-mail: xingzhao@gmc.edu.cn

Professor Zhixu He, National Joint Local Engineering Laboratory for Cell Engineering and Biomedicine Technique, Guizhou Province Key Laboratory of Regenerative Medicine, Key Laboratory of Adult Stem Cell Translational Research, Chinese Academy of Medical Sciences, 9 Beijing Road, Guiyang, Guizhou 550004, P.R. China

E-mail: hzx@gmc.edu.cn

Key words: SARS-CoV-2, COVID-19, cytokine storm, immunoregulatory therapy strategies

\author{
2. SARS-CoV-2 and COVID-19 \\ 3. Cytokine storm \\ 4. Targeted cytokine storm therapy - the fight against \\ COVID-19 \\ 5. Conclusions and perspectives
}

\section{Introduction}

At the end of 2019, a coronavirus pneumonia pandemic emerged in Wuhan, China (1). Subsequent genome sequencing and phylogenetic analyses revealed that this virus was a novel coronavirus. The International Committee on Taxonomy of Viruses designated it as severe acute respiratory syndrome coronavirus 2 (SARS-CoV-2), and the associated disease was named coronavirus disease 2019 (COVID-19) by the World Health Organization (WHO). As of August 30, 2020, there have been 24,854,140 confirmed cases of COVID-19 worldwide, including 838,924 deaths, and the disease has been listed as a public health emergency of international concern by the WHO (2). In a similar manner to severe acute respiratory syndrome coronavirus (SARS-CoV) and Middle East respiratory syndrome coronavirus (MERS-CoV), COVID-19 presents with severe respiratory syndrome $(3,4)$. However, researchers are currently attempting to identify treatments that suppress the transmission of SARS-CoV-2 or ameliorate the symptoms of COVID-19 (5). Considerable evidence from preclinical and clinical studies indicates that cytokine storm syndrome may be an important mechanism underlying this respiratory syndrome (6). An imbalance in immune regulation leads to an overwhelming release of cytokines, which is more harmful to the body than SARS-CoV-2 itself (7). In the present review, the progress of preclinical and clinical studies is summarized, and immunomodulatory therapies for patients with COVID-19 are reviewed and discussed.

\section{SARS-CoV-2 and COVID-19}

SARS-CoV-2 is an enveloped coronavirus that contains a single-stranded RNA genome. The particles are 50-200 nm in diameter, and comprise three envelope glycoproteins: Spike 
protein (S protein), envelope protein (E protein) and membrane protein (M protein). The $\mathrm{S}$ protein is closely associated with the ability of SARS-CoV-2 to invade cells, and is an important antigen in vaccine research and development in terms of the neutralization of antibody production (8). The M protein is transmembrane in nature, and plays an important role in envelope formation and virus budding (9). The E protein is mainly distributed over the capsule. The nucleocapsid phosphoprotein (N protein) is another important structural protein, which binds to viral RNA, forming the core.

According to several systematic analyses of COVID-19 cases (10-12), the most common clinical symptoms are fever, dry cough, myalgia or fatigue, with other symptoms including sputum production, headaches and hemoptysis. Some patients exhibit other systemic symptoms, including diarrhea and other digestive system ailments (10-12). However, other severe symptoms with the potential to become life-threatening may become manifest, including dyspnea, which can rapidly develop into acute respiratory distress syndrome (ARDS), arrhythmia, shock and multiple organ dysfunction syndrome (MODS) (4,10-12). The mechanism of SARS-CoV-2 infection is believed to occur via viral binding to host cell surface receptors, specifically angiotensin-converting enzyme 2 (ACE2) receptors (13). ACE2 receptors are distributed over arteriovenous endothelial cells, arterial smooth muscle cells, intestinal epithelial cells, alveoli, bronchi and other respiratory organs. The virus infects these cells by binding to ACE2 receptors, causing pathological changes in corresponding organs, the respiratory and digestive systems, heart and nervous system $(14,15)$.

\section{Cytokine storm}

Cytokine storm, also known as inflammatory storm or cytokine release syndrome, refers to an excessive immune response elicited by viruses, bacteria or other external stimuli (16). During a cytokine storm, cytokines such as tumor necrosis factor (TNF)- $\alpha$, interleukin (IL)-1, IL-6, IL-8, IL-12, interferon (IFN)- $\alpha$, IFN- $\gamma$ and monocyte chemotactic protein-1 (MCP-1) are rapidly produced in the host. Excessively secreted cytokines facilitate the chemotaxis of neutrophils, monocytes and eosinophils to inflammatory sites, where they not only clear viral particles but also may cause organ failure. Importantly, these cytokines also activate immune cells, further increasing the production of cytokines. Under physiological conditions, feedback loops, precisely controlled by anti-inflammatory cytokines, are provided by $\mathrm{T}$ helper type 2 cells and regulatory $\mathrm{T}$ cells (Tregs). However, during pathological conditions, where highly pathogenic viral particles are present, these feedback loops are imbalanced and cause positive feedback that results in the amplification of cytokine production. This suicidal immune response is the cause of ARDS and MODS, which may eventually culminate in death (17).

Previous studies on SARS-CoV, MERS-CoV, influenza A H1N1 virus, avian influenza virus and other viruses have suggested that cytokine storms are primary mechanisms leading to fatal pneumonia. Xu et al (18) detected cytokines in the blood of patients with SARS, and revealed that inflammatory factors, mainly represented by IFN- $\gamma$-inducible protein 10 (IP-10), were highly elevated. This cytokine damages vascular endothelial and respiratory epithelial cells, culminating in ARDS (18). Another study showed that large numbers of serum-based pro-inflammatory cytokines, including IL-1 $\beta$, IL-6, IFN- $\gamma$, IP-10 and MCP-1, are involved in the extensive lung injury mediated by SARS-CoV (19). In an analysis of the cytokine response to MERS-CoV in patient plasma, Mahallawi et al (20) identified prominent pro-inflammatory $\mathrm{T}$ helper (Th)1 and Th17 responses and markedly increased secretion of the cytokines IFN- $\gamma$, TNF- $\alpha$, IL-15 and IL-17. These data suggest a significant pro-inflammatory cytokine response to the acute phase of MERS-CoV infection, which may be associated with disease severity. Importantly, SARS-CoV-2 infections also generate cytokine storm events similar to those induced by SARS-CoV and MERS-CoV. Huang et al (12) analyzed 41 patients with COVID-19 and found that $63 \%$ had lymphocytopenia, with markedly increased concentrations of IL-2, IL-7, IL-10, granulocyte colony stimulating factor (G-CSF), IP-10, MCP-1, macrophage inflammatory protein $1 \mathrm{~A}$ and TNF- $\alpha$ in intensive care unit (ICU) patients, when compared with non-ICU patients. Thus, cytokine storms may be an important contributor to the pathogenesis of COVID-19. Initially, SARS-CoV-2 infection leads to a loss of negative feedback in the regulation of the immune response, which abnormally increases the levels of certain cytokines. This increase promotes the recruitment and activation of immune cells, which cause diffuse damage to pulmonary capillary endothelial and alveolar epithelial cells, leading to ARDS and possibly also MODS (21).

Although there are as yet no specific treatments for the cytokine storms induced by SARS-CoV-2, several non-specific treatments have been investigated since the pandemic outbreak. The present review discusses these treatment strategies for cytokine storms in patients with COVID-19.

\section{Targeted cytokine storm therapy - the fight against COVID-19}

Monoclonal antibodies. The excessive secretion of IL-6 $(>1,000 \mathrm{pg} / \mathrm{ml}$ in serum) can lead to vascular leakage, tissue hypoxia, hypotension and myocardial dysfunction, resulting in MODS and disseminated intravascular coagulation (22). Additionally, IL-6 reduces the release of perforin and granzymes from natural killer (NK) cells and impairs their antiviral activity (23). During a cytokine storm, the duration of elevated IL-6 secretion is longer than that of other cytokines, suggesting that inhibition of IL-6 or its receptor (IL-6R) could be a viable therapeutic strategy during a SARS-CoV-2 infection $(24,25)$.

A number of studies have confirmed the safety and efficacy of the anti-IL-6 antibody siltuximab, and the anti-IL-6R antibodies tocilizumab and sarilumab (25-27). To date, more than 40 clinical trials using anti-IL-6 or anti-IL-6R treatments have commenced for patients with COVID-19, including more than 30 trials using tocilizumab (Table I). This humanized monoclonal antibody inhibits IL-6R by blocking the binding of IL-6 to its receptor and inhibiting its signaling (28). In the past, tocilizumab was primarily used in the treatment of cytokine storms caused by chimeric antigen receptor T cell (CAR-T) therapy (29). In one study, the levels of IL-6, IL-8 and IL-10 were observed to be elevated to varying 


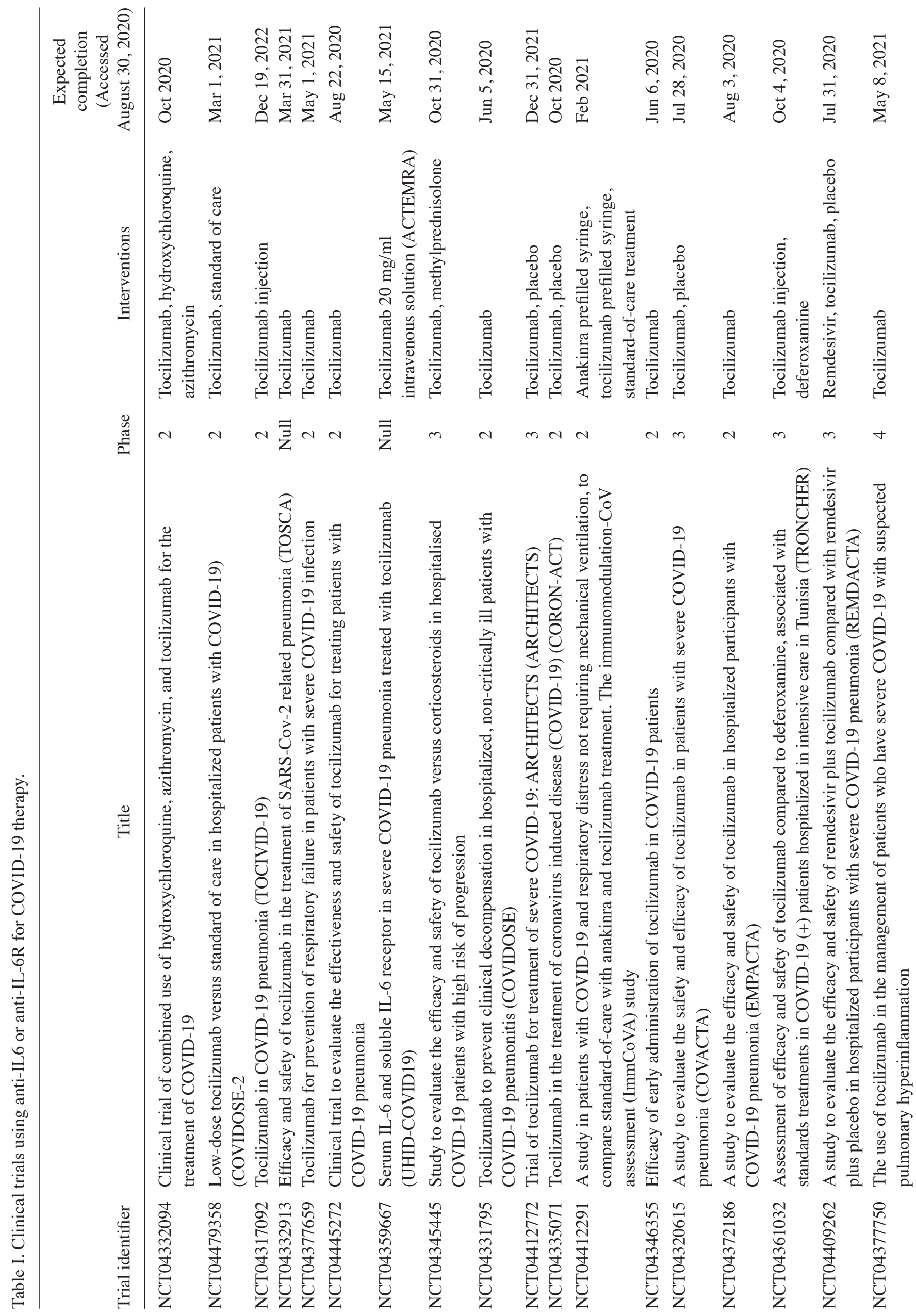




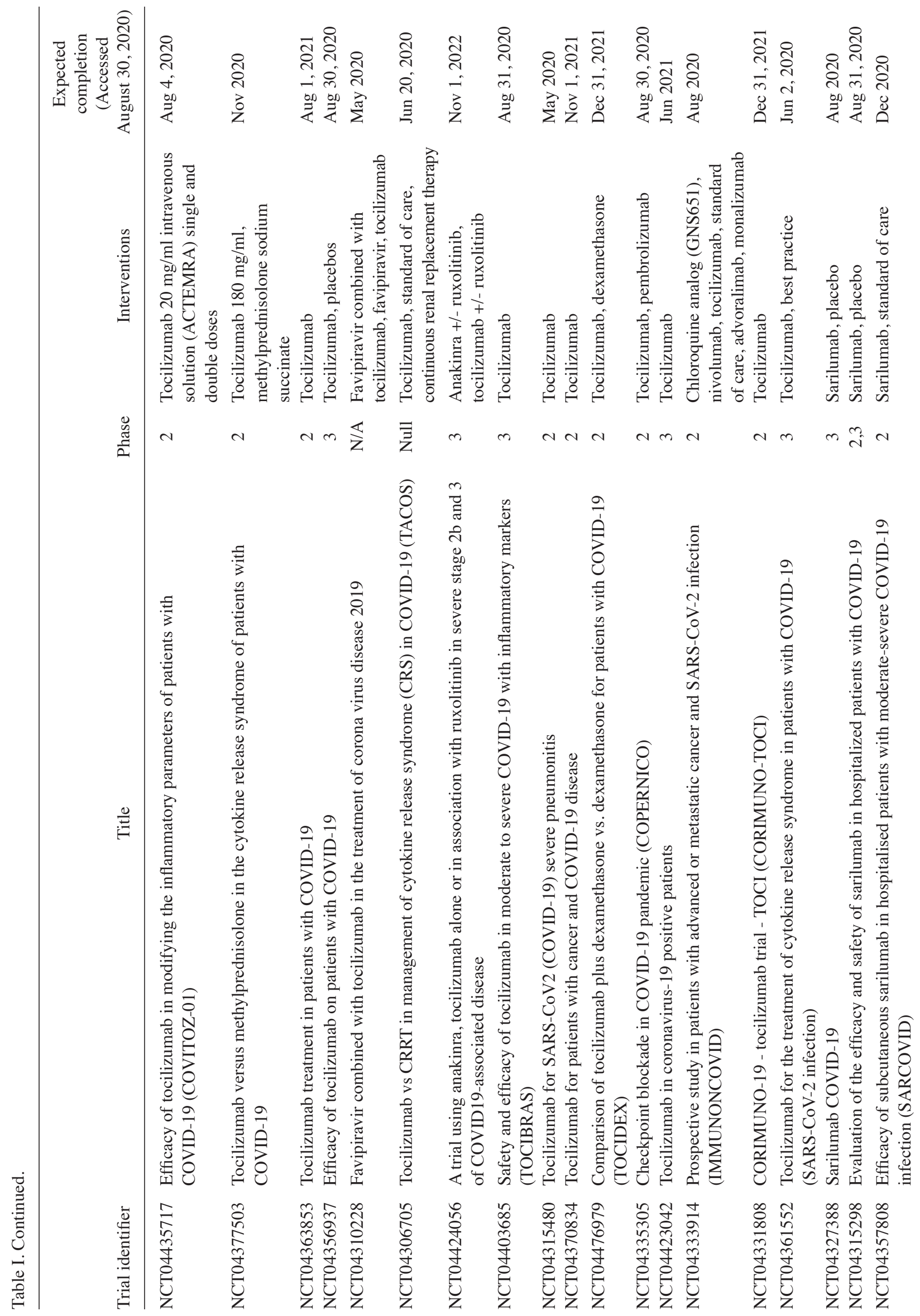




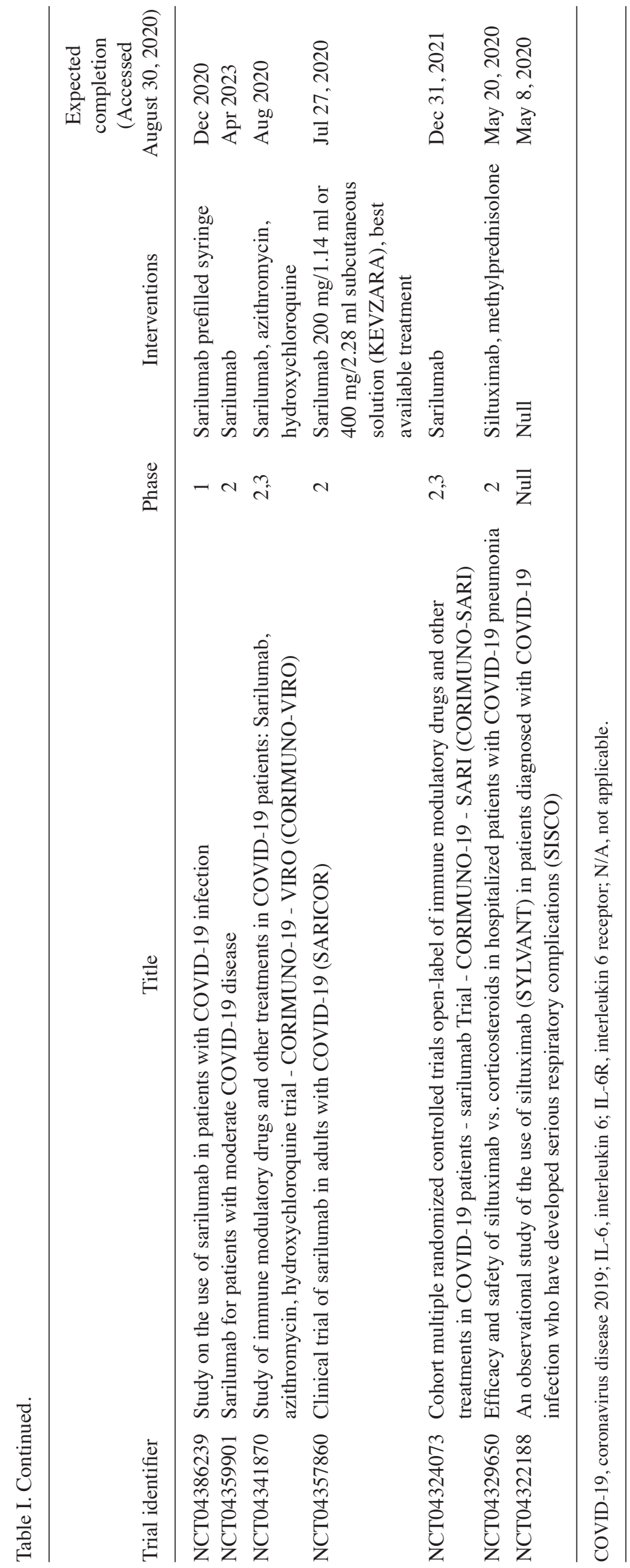


degrees in four cases of CAR-T-induced cytokine storm. However, after treatment with tocilizumab, the symptoms of systemic toxicity were significantly ameliorated, the cytokine levels decreased, and the requirements for adjuvant and other therapies, including vasoactive drugs, glucocorticoids and respiratory support, were reduced (30). Tocilizumab has also been observed to attenuate the excessive production of other cytokines, namely IFN- $\gamma$, IL-10 and IL-2, and the expansion of cytotoxic T and NK cells in refractory hemophagocytic lymphohistiocytosis (31). Retrospective Chinese studies have also confirmed the positive effects of tocilizumab in severe or critical cases of COVID-19 $(32,33)$. However, as patient numbers were low in these studies, the clinical efficacy of tocilizumab requires further testing.

Recombinant granulocyte-macrophage colony stimulating factor. Alveolar type II epithelial cells accurately regulate the production of granulocyte-macrophage colony stimulating factor (GM-CSF), which activates innate and adaptive immune responses, and improves the ability of the body to fight against viruses (34). GM-CSF also stimulates the proliferation of alveolar epithelial cells in order to repair broken lung barriers, and protect the lungs from secondary bacterial infection following viral infection (35). SARS-CoV-2 infects type II alveolar epithelial cells via the ACE2 receptor, thereby destroying pulmonary physiological barriers and leading to imbalanced GM-CSF regulation (36). A recent study showed that G-CSF levels in the peripheral blood of patients with COVID-19 were elevated, particularly those critically ill in intensive care (12). It is speculated that blocking GM-CSF or using anti-GM-CSF drugs could be a viable treatment strategy for COVID-19. Notably, in recent months, more than 10 clinical trials have been initiated using this strategy (Table II).

$I F N$. As a major effector cytokine of the host immune response to viral infection, IFN serves as an immunomodulator by promoting macrophage-mediated antigen phagocytosis, and mediating the clearance of infected cells by NK cells, thus limiting viral transmission. IFNs are also often used to treat viral diseases such as hepatitis B and C (37). Evidence from preclinical and clinical studies indicates that the earlier IFN production occurs after coronavirus infection, the less viral replication occurs and the lower the mortality rate $(38,39)$. Chu et al (40) compared immune activation between the lung tissues of patients with SARS-CoV-2 and SARS-CoV infections, and found that the lungs infected with SARS-CoV-2 did not produce elevated quantities of IFNs. Therefore, it is suggested that exogenous IFN should be administered to stimulate host antiviral immunity in patients infected with SARS-CoV-2. In addition, IFN- $\lambda$ has been demonstrated to reduce the risk of SARS-CoV-2 transmission and the severity of COVID-19 (41).

Mesenchymal stem cell (MSC)-based therapy. MSCs are a type of non-hematopoietic stem cell, derived from several tissues, including Wharton's jelly, umbilical cord blood, placenta, bone marrow, adipose tissue, dental pulp and menstrual blood (42). Evidence from preclinical and clinical studies has confirmed that MSCs function by promoting tissue regeneration and protecting against injury through self-renewal, multiple differentiation and paracrine functions $(43,44)$. Importantly, MSCs also exert strong immunomodulatory functions (45). Studies have shown that these cells regulate the activation, proliferation and differentiation of NK cells, dendritic cells, B and $\mathrm{T}$ lymphocytes, and other immune cells, and also increase the proportion of Tregs, thus maintaining immune system stability $(42,46,47)$. MSCs interact with immune cells, and potentially inhibit localized immune responses via the secretion of regulatory factors, including transforming growth factor $\beta$, hepatocyte growth factor and IL-10 (48). The immunomodulatory properties of MSCs have been shown to be effective in acute graft-versus-host disease (49), type 1 diabetes (50), rheumatoid arthritis (51), systemic lupus erythematosus (52), inflammatory bowel disease (53) and other immune and inflammation-associated diseases (54). The cells attenuate acute lung injury by inhibiting the infiltration of immune cells and reducing the secretion of inflammatory factors. Therefore, the immunomodulatory functions of MSCs may be effective in reducing the occurrence of cytokine storms in severe cases of COVID-19 (55). Indeed, at least 20 COVID-19 clinical trials using MSCs are ongoing (Table III). Although most preclinical studies on the immune effects of MSCs have shown benefits, further studies are required to evaluate the safety of MSC transplantation, particularly with regard to potential tumorigenic effects (56).

Thymosin. Thymosin induces T-cell differentiation, proliferation and maturation (57). In addition, it promotes the production of IL-2, thereby inducing anti-inflammatory effects (58). As an immune enhancer, thymosin is widely used in the adjuvant treatment of hepatitis (59), autoimmune diseases (60) and several types of tumors (61). The pathology report of a COVID-19 patient revealed that the numbers of $\mathrm{CD} 4^{+}$and $\mathrm{CD} 8^{+} \mathrm{T}$ cells in the peripheral blood were significantly decreased, and it was suggested that lymphopenia may be associated with severity disease and mortality (62). Therefore, thymosin may be useful in contributing to the reconstruction of effective T-cell immunity in patients with COVID-19, thereby potentially inhibiting cytokine storms. However, the safety and validity of thymosin in the treatment of COVID-19 requires investigation in clinical trials.

Immunoglobulin. Intravenous immunoglobulin (IVIG) preparations can neutralize antigens, and also regulate cytokine responses and immune cell functions. A retrospective study of 15 patients with severe sepsis reported that treatment with IgM-enriched immunoglobulins decreased endotoxin activity and ameliorated platelet loss and fibrinogen depletion (63). Another retrospective observational study evaluated the effects of IVIG in patients with bacterial or septic shocks, including 17 trials in adults and 8 in newborn infants (64). IVIG significantly reduced the mortality rates in adult sepsis, but not in neonatal sepsis. Notably, Cao et al (65) reported on three patients with severe COVID-19 who were treated with high-dose IVIG during an ARDS attack; following the treatment, their clinical conditions and associated laboratory and imaging examinations were improved, suggesting that a high-dose of IVIG in the early stages of clinical deterioration is able to prevent disease progression and improve the prognosis of COVID-19. 


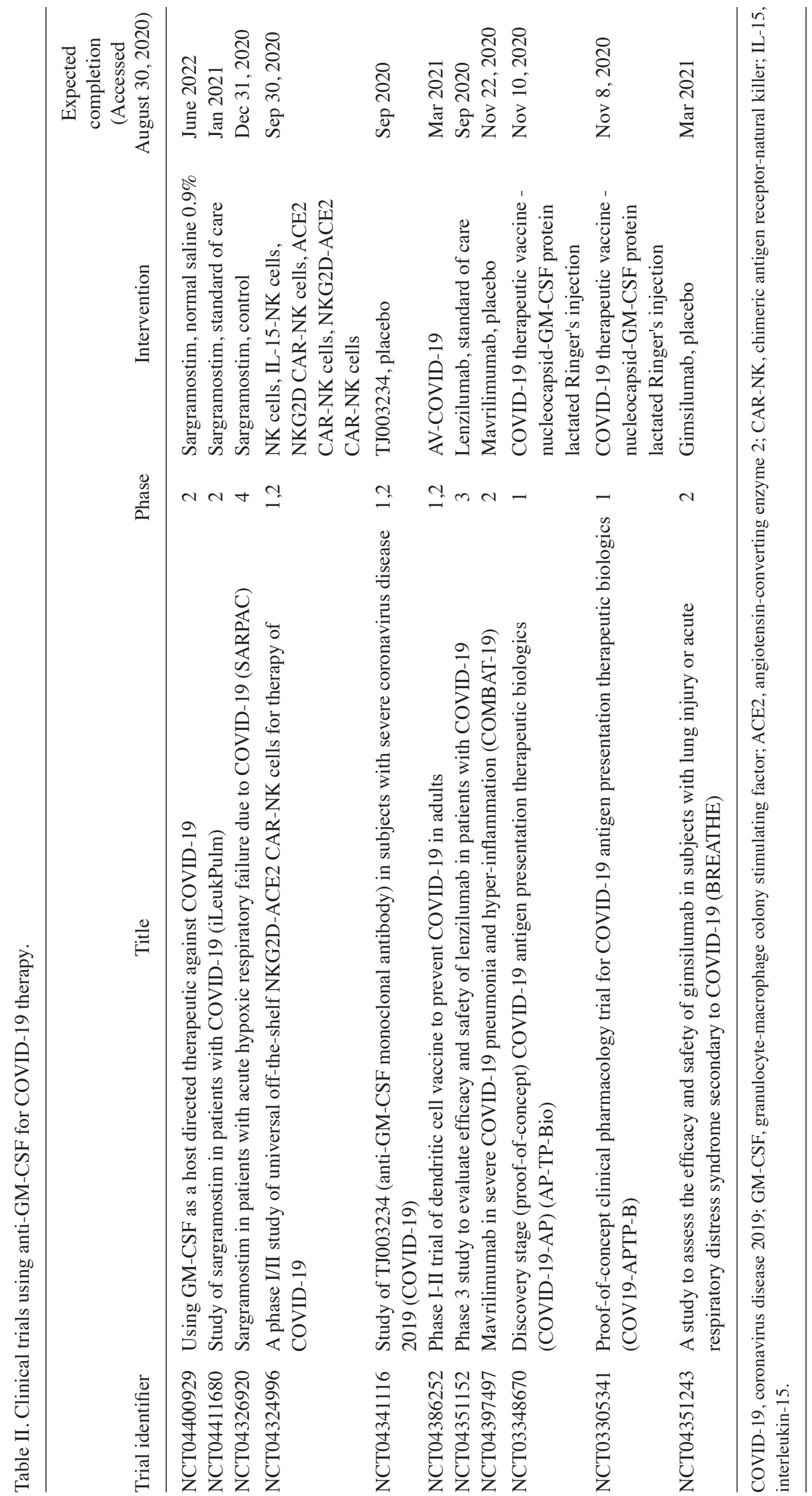




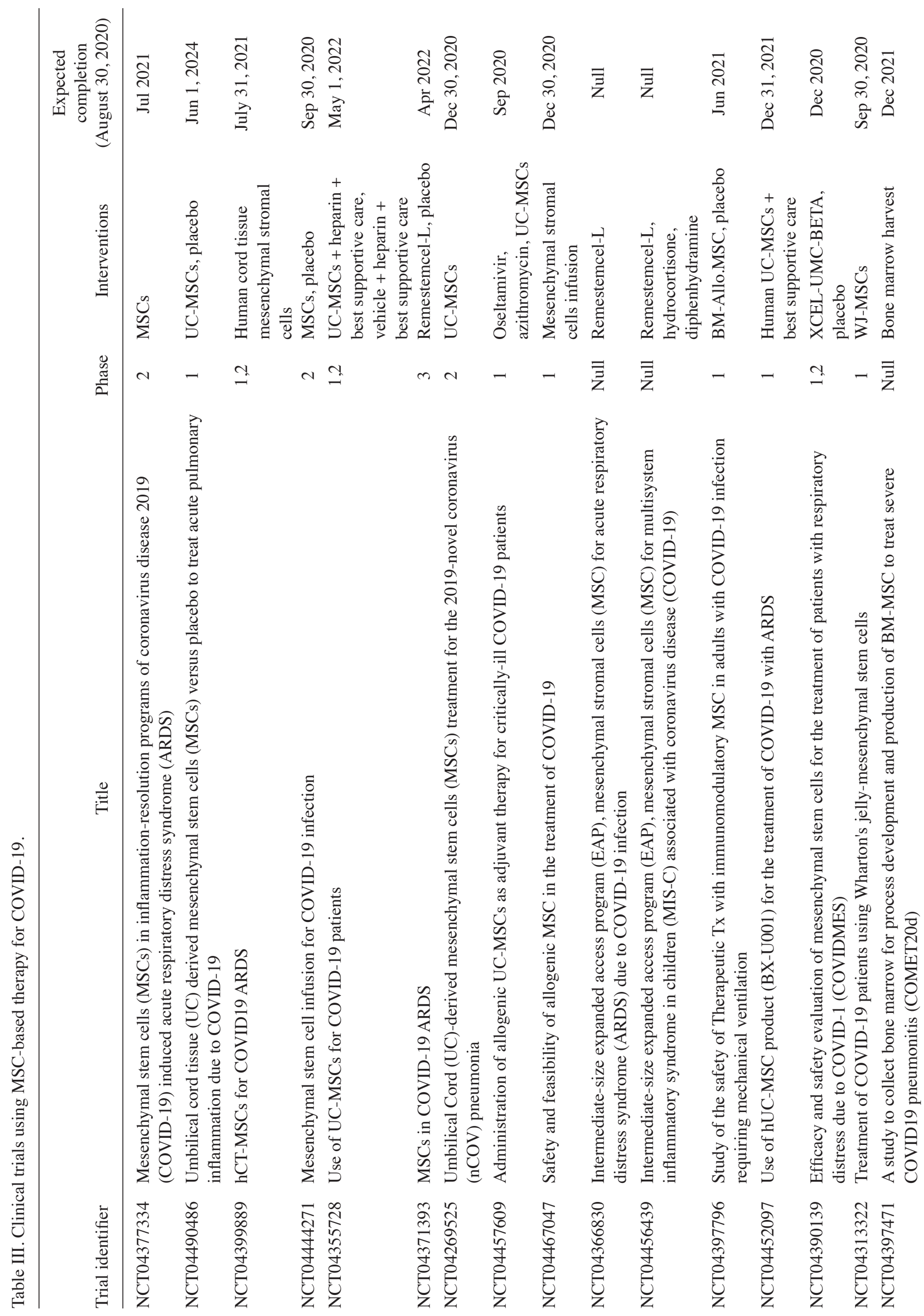


Blood purification therapy. A newly developed, non-specific, broad-spectrum blood purification therapy may also have applications in the targeting of cytokine storms during COVID-19 infections. Hemodialysis, hemofiltration, plasma exchange and hemoperfusion are four classical blood purification techniques used to combat drug poisoning, renal failure, multiple organ failure and septicemia (66). A retrospective Chinese study evaluated three patients who were diagnosed with severe or critical COVID-19 and treated with plasma exchange (67). The rate of plasma separation and infusion was $25-30 \mathrm{ml} / \mathrm{min}$, and the volume of each plasma exchange was 2,600-3,000 $\mathrm{ml}(67)$. The authors reported that this therapy significantly decreased C-reactive protein and IL-6 levels, and improved lymphocyte and prothrombin times, suggesting that it is a viable treatment for patients with severe COVID-19. However, a prospective observational study evaluated the efficacy of blood purification in 9 patients with sepsis/septic shock (68). After blood purification treatment, except for the plasma levels of IL-8 decrease, the level of other cytokines did not vary significantly, such as TNF- $\alpha$, IL- $1 \beta$, IL- 6 and IL10 (68). Therefore, further investigations are required to explore the clinical benefits of blood purification for cytokine storms induced by SARS-CoV-2 infection. Similarly, other factors for this therapy, such as the appropriate model, timing, course and frequency of treatment require investigation.

Others. During coronavirus-mediated pneumonia, the massive release of cytokines is an imbalanced antiviral immune response that can lead to life-threatening ARDS. Glucocorticoids exert anti-inflammatory, anti-toxic, anti-allergic and anti-shock effects $(69,70)$. A morbidly obese COVID-19 patient with urticaria and angioedema was successfully treated with glucocorticoids (70). However, long-term and high-dose use of glucocorticoid can cause secondary infection, osteonecrosis, diabetes and hypertension (71). Therefore, the timing of administration, dosage and treatment course require extensive clinical exploration.

The glycoproteins of coronavirus facilitate viral entry into target cells by binding to receptors and by driving fusion of viral and host cell membranes. However, the host cell protease activity determines the efficiency of glycoprotein synthesis (72). A recent study used a panel of cell lines to verify that ACE2 and transmembrane protease serine 2 (TMPRSS2) proteins are required for the infection of cells by SARS-CoV-2, similarly to SARS-CoV infection (73). TMPRSS2 inhibitors blocked the entry of SARS-CoV-2 into the cells, and thus displayed potential as antiviral inhibitors. Indeed, camostat mesylate, a serine protease inhibitor that inhibits TMPRSS2 protease activity, is widely used in Japan to alleviate acute inflammation during chronic pancreatitis (74). Therefore, this protease inhibitor may have therapeutic potential for the treatment of patients with COVID-19.

\section{Conclusion and perspectives}

The pathogenesis of COVID-19 resembles a prolonged battle between the virus and the immune system. When confronted by viral infection, the immune system must recognize and clear the virus in a timely manner. However, imbalanced and excessive immune responses may result 
in the excessive expression of inflammatory cytokines. Furthermore, these locally maladjusted immune responses may damage the oxygenation functions of the lungs, potentially resulting in MODS. Therefore, it is surmised that the excessive release of inflammatory cytokines may lead to severe COVID-19.

Antiviral, anti-inflammatory and organ-supporting therapies are considered to be the primary treatment strategies for patients with COVID-19. Antiviral therapies require antiviral drugs and vaccines; however, drug and vaccine development and preparation are challenging to achieve in the short term. Organ support therapy is an effective therapy in the treatment of severe COVID-19 patients with respiratory failure or ARDS $(75,76)$. However, in the context of the COVID-19 pandemic, organ support therapy may not be a widely used treatment due to the resource constraints and availability problems (77). Currently, a plethora of experimental and conventional drugs are actively undergoing clinical trials for the treatment and prevention of COVID-19. Some of these drugs exert therapeutic efficacies that are associated with regulation of the immune system. In addition, since MSCs have an immunosuppressive effect, and associated clinical trial data have shown significant therapeutic efficacy in severe cases of COVID-19 (78-80), MSC-based therapy could be a promising strategy for the reduction of inflammatory cytokine release in these patients. Therefore, we hypothesize that immunoregulatory therapy is currently the most promising treatment for severe COVID-19, especially for the elderly patients or those with underlying diseases. However, the stage during the development of COVID-19 at which cytokine storms occur, and the incidence and mortality rates of patients who experience cytokine storms are not yet known. Therefore, evidence from immunoregulatory preclinical and clinical studies is required for further verification.

It must be noted that the key to solving the SARS-COV-2 pandemic is the emergence of a vaccine. The research community must continue to comprehensively explore immune response mechanisms in the pathogenesis of COVID-19, in order to clarify relevant targets and signaling pathways. In adopting this approach, the promotion and advancement of novel therapeutic drugs and vaccines is likely to occur, providing a solid scientific foundation for the clinical diagnosis and treatment of COVID-19.

\section{Acknowledgements}

Not applicable.

\section{Funding}

This work was supported by the Non Profit Central Research Institute Fund of Chinese Academy of Medical Science (grant nos. 2018PT31048 and 2019PT310013) and the Special Grant for Central Government Supporting Local Science and Technology Development [grant no. (2019) 4008].

\section{Availability of data and materials}

Not applicable.

\section{Authors' contributions}

XW wrote the original draft, edited and critically revised the manuscript. XZ and $\mathrm{ZH}$ contributed substantially to the writing of the manuscript, and critically revised and edited the manuscript. All authors substantially contributed to the conception, writing and revision of the manuscript. All authors read and approved the final manuscript.

\section{Ethics approval and consent to participate}

Not applicable.

\section{Patient consent for publication}

Not applicable.

\section{Competing interests}

The authors declare that they have no competing interests.

\section{References}

1. Lum LHW and Tambyah PA: Outbreak of COVID-19 - an urgent need for good science to silence our fears? Singapore Med J 61: 55-57, 2020.

2. World Health Organization: WHO Coronavirus Disease (COVID-19) Dashboard. https://covid19.who.int/. Accessed August 30, 2020.

3. Li Q, Guan X, Wu P, Wang X, Zhou L, Tong Y, Ren R, Leung KSM, Lau EHY, Wong JY, et al: Early Transmission Dynamics in Wuhan, China, of Novel Coronavirus-Infected Pneumonia. N Engl J Med 382: 1199-1207, 2020.

4. Tsatsakis A, Calina D, Falzone L, Petrakis D, Mitrut R, Siokas V, Pennisi M, Lanza G, Libra M, Doukas SG, et al: SARS-CoV-2 pathophysiology and its clinical implications: An integrative overview of the pharmacotherapeutic management of COVID-19. Food Chem Toxicol 146: 111769, 2020.

5. Nitulescu GM,Paunescu H, Moschos SA, Petrakis D, Nitulescu G, Ion GND, Spandidos DA, Nikolouzakis TK, Drakoulis N and Tsatsakis A: Comprehensive analysis of drugs to treat SARS CoV 2 infection: Mechanistic insights into current COVID 19 therapies (Review). Int J Mol Med 46: 467-488, 2020.

6. Ruscitti P, Berardicurti O, Iagnocco A and Giacomelli R: Cytokine storm syndrome in severe COVID-19. Autoimmun Rev 19: 102562, 2020.

7. Napp LC and Bauersachs J: Extracorporeal Hemoadsorption: An Option for COVID-19-Associated Cytokine Storm Syndrome. Shock 54: 700-701, 2020.

8. Liu L, Wang P, Nair MS, Yu J, Rapp M, Wang Q, Luo Y, Chan JF, Sahi V, Figueroa A, et al: Potent neutralizing antibodies against multiple epitopes on SARS-CoV-2 spike. Nature 584: 450-456, 2020.

9. Satarker S and Nampoothiri M: Structural Proteins in Severe Acute Respiratory Syndrome Coronavirus-2. Arch Med Res 51: 482-491, 2020.

10. Chen N, Zhou M, Dong X, Qu J, Gong F, Han Y, Qiu Y, Wang J, Liu Y, Wei Y, et al: Epidemiological and clinical characteristics of 99 cases of 2019 novel coronavirus pneumonia in Wuhan, China: A descriptive study. Lancet 395: 507-513, 2020.

11. Wang D, Hu B, Hu C, Zhu F, Liu X, Zhang J, Wang B, Xiang H, Cheng Z, Xiong Y, et al: Clinical Characteristics of 138 Hospitalized Patients With 2019 Novel Coronavirus-Infected Pneumonia in Wuhan, China. JAMA 323: 1061-1069, 2020.

12. Huang C, Wang Y, Li X, Ren L, Zhao J, Hu Y, Zhang L, Fan G, $\mathrm{Xu} \mathrm{J}, \mathrm{Gu} \mathrm{X}$, et al: Clinical features of patients infected with 2019 novel coronavirus in Wuhan, China. Lancet 395: 497-506, 2020.

13. Wan Y, Shang J, Graham R, Baric RS and Li F: Receptor Recognition by the Novel Coronavirus from Wuhan: An Analysis Based on Decade-Long Structural Studies of SARS Coronavirus. J Virol 94: e00127-e20, 2020. 
14. Mao L, Jin H, Wang M, Hu Y, Chen S, He Q, Chang J, Hong C, Zhou Y, Wang D, et al: Neurologic Manifestations of Hospitalized Patients With Coronavirus Disease 2019 in Wuhan, China. JAMA Neurol 77: 683-690, 2020.

15. Xu X, Chen P, Wang J, Feng J, Zhou H, Li X, Zhong W and Hao P: Evolution of the novel coronavirus from the ongoing Wuhan outbreak and modeling of its spike protein for risk of human transmission. Sci China Life Sci 63: 457-460, 2020.

16. Mangalmurti $\mathrm{N}$ and Hunter CA: Cytokine Storms: Understanding COVID-19. Immunity 53: 19-25, 2020.

17. Lin SH, Zhao YS, Zhou DX, Zhou FC and Xu F: Coronavirus disease 2019 (COVID-19): Cytokine storms, hyper-inflammatory phenotypes, and acute respiratory distress syndrome. Genes Dis 7: $520-527,2020$

18. Xu J, Zhong S, Liu J, Li L, Li Y, Wu X, Li Z, Deng P, Zhang J, Zhong N, et al: Detection of severe acute respiratory syndrome coronavirus in the brain: Potential role of the chemokine mig in pathogenesis. Clin Infect Dis 41: 1089-1096, 2005.

19. Wong CK, Lam CW, Wu AK, Ip WK, Lee NL, Chan IH, Lit LC, Hui DS, Chan MH, Chung SS, et al: Plasma inflammatory cytokines and chemokines in severe acute respiratory syndrome. Clin Exp Immunol 136: 95-103, 2004.

20. Mahallawi WH, Khabour OF, Zhang Q, Makhdoum HM and Suliman BA: MERS-CoV infection in humans is associated with a pro-inflammatory Th1 and Th17 cytokine profile. Cytokine 104: 8-13, 2018.

21. Neurath MF: COVID-19 and immunomodulation in IBD. Gut 69 $1335-1342,2020$

22. Sapan HB, Paturusi I, Jusuf I, Patellongi I, Massi MN Pusponegoro AD, Arief SK, Labeda I, Islam AA, Rendy L, et al: Pattern of cytokine (IL-6 and IL-10) level as inflammation and anti-inflammation mediator of multiple organ dysfunction syndrome (MODS) in polytrauma. Int J Burns Trauma 6: 37-43, 2016.

23. Kang YJ, Jeung IC, Park A, Park YJ, Jung H, Kim TD, Lee HG Choi I and Yoon SR: An increased level of IL-6 suppresses NK cell activity in peritoneal fluid of patients with endometriosis via regulation of SHP-2 expression. Hum Reprod 29: 2176-2189, 2014.

24. Wang C, Fei D, Li X, Zhao M and Yu K: IL-6 may be a good biomarker for earlier detection of COVID-19 progression. Intensive Care Med 46: 1475-1476, 2020.

25. Crisafulli S, Isgrò V, La Corte L, Atzeni $F$ and Trifirò $G$ : Potential Role of Anti-interleukin (IL)-6 Drugs in the Treatment of COVID-19: Rationale, Clinical Evidence and Risks. BioDrugs 34: 415-422, 2020

26. Velasco Puyo P, Moreno L, Diaz de Heredia C, Riviere JG and Soler Palacin P: Tocilizumab in a child with acute lymphoblastic leukaemia and COVID-19-related cytokine release syndrome. An Pediatr (Engl Ed) 93: 132-133, 2020.

27. Qian S, An J, Qi F, Ye L, Chen Q, Liu X, Xie L and Li G: Tocilizumab exerts anti-inflammatory activity in six critically il COVID-19 patients: A retrospective analysis. Ann Transl Med 8 : $881,2020$.

28. Tanaka T, Narazaki $M$ and Kishimoto T: Immunotherapeutic implications of IL-6 blockade for cytokine storm. Immunotherapy 8: 959-970, 2016.

29. Le RQ, Li L, Yuan W, Shord SS, Nie L, Habtemariam BA, Przepiorka D, Farrell AT and Pazdur R: FDA Approval Summary: Tocilizumab for Treatment of Chimeric Antigen Receptor T Cell-Induced Severe or Life-Threatening Cytokine Release Syndrome. Oncologist 23: 943-947, 2018.

30. Chen H, Wang F, Zhang P, Zhang Y, Chen Y, Fan X, Cao X, Liu J, Yang Y, Wang B, et al: Management of cytokine release syndrome related to CAR-T cell therapy. Front Med 13: 610-617, 2019.

31. Faguer S, Vergez F, Peres M, Ferrandiz I, Casemayou A, Belliere J, Cointault O, Lavayssiere L, Nogier MB, Prevot G, et al: Tocilizumab added to conventional therapy reverses both the cytokine profile and $\mathrm{CD} 8^{+} \mathrm{Granzyme}^{+} \mathrm{T}$-cells/NK cells expansion in refractory hemophagocytic lymphohistiocytosis. Hematol Oncol 34: 55-57, 2016

32. Luo P, Liu Y, Qiu L, Liu X, Liu D and Li J: Tocilizumab treatment in COVID-19: A single center experience. J Med Virol 92: 814-818, 2020

33. Xu X, Han M, Li T, Sun W, Wang D, Fu B, Zhou Y, Zheng X, Yang Y, Li X, et al: Effective treatment of severe COVID-19 patients with tocilizumab. Proc Natl Acad Sci USA 117: 10970-10975, 2020

34. Carey B and Trapnell BC: The molecular basis of pulmonary alveolar proteinosis. Clin Immunol 135: 223-235, 2010.
35. Sever-Chroneos Z, Murthy A, Davis J, Florence JM, Kurdowska A, Krupa A, Tichelaar JW, White MR, Hartshorn KL, Kobzik L, et al: GM-CSF modulates pulmonary resistance to influenza A infection. Antiviral Res 92: 319-328, 2011.

36. Damiani G, McCormick TS, Leal LO and Ghannoum MA Recombinant human granulocyte macrophage-colony stimulating factor expressed in yeast (sargramostim): A potential ally to combat serious infections. Clin Immunol 210: 108292, 2020.

37. Li SF, Gong MJ, Zhao FR, Shao JJ, Xie YL, Zhang YG and Chang HY: Type I Interferons: Distinct Biological Activities and Current Applications for Viral Infection. Cell Physiol Biochem 51: 2377-2396, 2018.

38. González-Navajas JM, Lee J, David $M$ and Raz E: Immunomodulatory functions of type I interferons. Nat Rev Immunol 12: 125-135, 2012

39. Wang N, Zhan Y, Zhu L, Hou Z, Liu F, Song P, Qiu F, Wang X, Zou X, Wan D, et al: Retrospective Multicenter Cohort Study Shows Early Interferon Therapy Is Associated with Favorable Clinical Responses in COVID-19 Patients. Cell Host Microbe 28 455-464.e2, 2020.

40. Chu H, Chan JF, Wang Y, Yuen TT, Chai Y, Hou Y, Shuai H, Yang D, Hu B, Huang X, et al: Comparative replication and immune activation profiles of SARS-CoV-2 and SARS-CoV in human lungs: An ex vivo study with implications for the pathogenesis of COVID-19. Clin Infect Dis 71: 1400-1409, 2020.

41. O'Brien TR, Thomas DL, Jackson SS, Prokunina-Olsson L, Donnelly RP and Hartmann R: Weak Induction of Interferon Expression by SARS-CoV-2 Supports Clinical Trials of Interferon Lambda to Treat Early COVID-19. Clin Infect Dis 71: 1410-1412, 2020.

42. Naji A, Eitoku M, Favier B, Deschaseaux F, Rouas-Freiss N and Suganuma N: Biological functions of mesenchymal stem cells and clinical implications. Cell Mol Life Sci 76: 3323-3348, 2019.

43. Enciso N, Avedillo L, Fermín ML, Fragío C and Tejero C: Regenerative potential of allogeneic adipose tissue-derived mesenchymal cells in canine cutaneous wounds. Acta Vet Scand 62: 13, 2020.

44. Wang X, Wang H, Lu J, Feng Z, Liu Z, Song H, Wang H, Zhou Y and Xu J: Erythropoietin-Modified Mesenchymal Stem Cells Enhance Anti-fibrosis Efficacy in Mouse Liver Fibrosis Model. Tissue Eng Regen Med 17: 683-693, 2020.

45. Baharlou R, Rashidi N, Ahmadi-Vasmehjani A, Khoubyari M, Sheikh M and Erfanian S: Immunomodulatory Effects of Human Adipose Tissue-derived Mesenchymal Stem Cells on T Cell Subsets in Patients with Rheumatoid Arthritis. Iran J Allergy Asthma Immunol 18: 114-119, 2019.

46. Corcione A, Benvenuto F, Ferretti E, Giunti D, Cappiello V, Cazzanti F, Risso M, Gualandi F, Mancardi GL, Pistoia V, et al: Human mesenchymal stem cells modulate B-cell functions. Blood 107: 367-372, 2006

47. Luz-Crawford P, Kurte M, Bravo-Alegría J, Contreras R, Nova-Lamperti E, Tejedor G, Noël D, Jorgensen C, Figueroa F, Djouad F, et al: Mesenchymal stem cells generate a CD $4^{+} \mathrm{CD} 25^{+} \mathrm{Foxp}^{+}$regulatory $\mathrm{T}$ cell population during the differentiation process of Th1 and Th17 cells. Stem Cell Res Ther 4: 65, 2013 .

48. Gao F, Chiu SM, Motan DA, Zhang Z, Chen L, Ji HL, Tse HF, Fu QL and Lian Q: Mesenchymal stem cells and immunomodulation: Current status and future prospects. Cell Death Dis 7: e2062, 2016.

49. Elgaz S, Kuçi Z, Kuçi S, Bönig H and Bader P: Clinical Use of Mesenchymal Stromal Cells in the Treatment of Acute Graft-versus-Host Disease. Transfus Med Hemother 46: 27-34, 2019.

50. Kamal MM and Kassem DH: Therapeutic Potential of Wharton's Jelly Mesenchymal Stem Cells for Diabetes: Achievements and Challenges. Front Cell Dev Biol 8: 16, 2020.

51. Yang JH, Liu FX, Wang JH, Cheng M, Wang SF and Xu DH: Mesenchymal stem cells and mesenchymal stem cell-derived extracellular vesicles: Potential roles in rheumatic diseases. World J Stem Cells 12: 688-705, 2020.

52. Radmanesh F, Mahmoudi M, Yazdanpanah E, Keyvani V, Kia N, Nikpoor AR, Zafari P and Esmaeili SA: The immunomodulatory effects of mesenchymal stromal cell-based therapy in human and animal models of systemic lupus erythematosus. IUBMB Life 72: 2366-2381, 2020

53. Fu Y, Ni J, Chen J, Ma G, Zhao M, Zhu S, Shi T, Zhu J, Huang Z, Zhang J, et al: Dual-Functionalized MSCs that Express CX3CR1 and IL-25 Exhibit Enhanced Therapeutic Effects on Inflammatory Bowel Disease. Mol Ther 28: 1214-1228, 2020. 
54. Regmi S, Pathak S, Kim JO, Yong CS and Jeong JH: Mesenchymal stem cell therapy for the treatment of inflammatory diseases: Challenges, opportunities, and future perspectives. Eur J Cell Biol 98: 151041, 2019.

55. Wang XY: MSCs transplantation may be a potential therapeutic strategy for COVID-19 treatment. Eur Rev Med Pharmacol Sci 24: 4537-4538, 2020.

56. Karaöz E and Tepeköy F: Differentiation Potential and Tumorigenic Risk of Rat Bone Marrow Stem Cells Are Affected By Long-Term In Vitro Expansion. Turk J Haematol 36: 255-265, 2019.

57. Shao C, Tian G, Huang Y, Liang W, Zheng H, Wei J, Wei C, Yang C, Wang $\mathrm{H}$ and Zeng W: Thymosin alpha-1-transformed Bifidobacterium promotes T cell proliferation and maturation in mice by oral administration. Int Immunopharmacol 15: 646-653, 2013.

58. Hsia J, Sarin N, Oliver JH and Goldstein AL: Aspirin and thymosin increase interleukin-2 and interferon-gamma production by human peripheral blood lymphocytes. Immunopharmacology 17 $167-173,1989$.

59. Wu X, Jia J and You H: Thymosin alpha-1 treatment in chronic hepatitis B. Expert Opin Biol Ther 15 (Suppl 1): S129-S132, 2015.

60. Goldstein AL, Zatz MM, Low TL and Jacobs R: Potential role of thymosin in the treatment of autoimmune diseases. Ann N Y Acad Sci 377 (1 Myasthenia Gr): 486-495, 1981.

61. Ioannou K, Samara P, Livaniou E, Derhovanessian E and Tsitsilonis OE: Prothymosin alpha: A ubiquitous polypeptide with potential use in cancer diagnosis and therapy. Cancer Immunol Immunother 61: 599-614, 2012.

62. Xu Z, Shi L, Wang Y, Zhang J, Huang L, Zhang C, Liu S, Zhao P, Liu H, Zhu L, et al: Pathological findings of COVID-19 associated with acute respiratory distress syndrome. Lancet Respir Med 8: 420-422, 2020

63. Wand S, Klages M, Kirbach C, Warszawska J, Meybohm P, Zacharowski $\mathrm{K}$ and Koch A: IgM-Enriched Immunoglobulin Attenuates Systemic Endotoxin Activity in Early Severe Sepsis: A Before-After Cohort Study. PLoS One 11: e0160907, 2016.

64. Alejandria MM, Lansang MA, Dans LF and Mantaring JB III: Intravenous immunoglobulin for treating sepsis, severe sepsis and septic shock. Cochrane Database Syst Rev 2013: CD001090, 2013.

65. Cao W, Liu X, Bai T, Fan H, Hong K, Song H, Han Y, Lin L, Ruan L and Li T: High-Dose Intravenous Immunoglobulin as a Therapeutic Option for Deteriorating Patients With Coronavirus Disease 2019. Open Forum Infect Dis 7: ofaa102, 2020.

66. Stegmayr B, Ramlow W and Balogun RA: Beyond dialysis: Current and emerging blood purification techniques. Semin Dial 25: 207-213, 2012.

67. Luo S, Yang L, Wang C, Liu C and Li D: Clinical observation of 6 severe COVID-19 patients treated with plasma exchange or tocilizumab. Zhejiang Da Xue Xue Bao Yi Xue Ban 49: 227-231, 2020 (In Chinese).

68. Zuccari S, Damiani E, Domizi R, Scorcella C, D'Arezzo M, Carsetti A,Pantanetti S, Vannicola S,CasarottaE, Ranghino A, et al: Changes in Cytokines, Haemodynamics and Microcirculation in Patients with Sepsis/Septic Shock Undergoing Continuous Renal Replacement Therapy and Blood Purification with CytoSorb. Blood Purif 49: 107-113, 2020.
69. Jiménez-Brítez G, Ruiz P and Soler X: Tocilizumab plus glucocorticoids in severe and critically COVID-19 patients. A single center experience. Med Clin (Barc) 155: 410-411, 2020 (In En, Spanish).

70. Lockey RF and Hudey SN: Coronavirus disease 2019-associated urticaria with angioedema in a morbidly obese man successfully treated with glucocorticoids. Ann Allergy Asthma Immunol 125: 359-360, 2020

71. Zhao R, Wang H, Wang X and Feng F: Steroid therapy and the risk of osteonecrosis in SARS patients: A dose-response meta-analysis. Osteoporos Int 28: 1027-1034, 2017.

72. Zhou Y, Vedantham P, Lu K, Agudelo J, Carrion R Jr, Nunneley JW, Barnard D, Pöhlmann S, McKerrow JH Renslo AR, et al: Protease inhibitors targeting coronavirus and filovirus entry. Antiviral Res 116: 76-84, 2015.

73. Hoffmann M, Kleine-Weber H, Schroeder S, Krüger N, Herrler T, Erichsen S, Schiergens TS, Herrler G, Wu NH, Nitsche A, et al: SARS-CoV-2 Cell Entry Depends on ACE2 and TMPRSS2 and Is Blocked by a Clinically Proven Protease Inhibitor. Cell 181: 271-280.e8, 2020.

74. Ramsey ML, Nuttall J, Hart PA; TACTIC Investigative Team: A phase $1 / 2$ trial to evaluate the pharmacokinetics, safety, and efficacy of NI-03 in patients with chronic pancreatitis: Study protocol for a randomized controlled trial on the assessment of camostat treatment in chronic pancreatitis (TACTIC). Trials 20: 501, 2019.

75. Hong X, Xiong J, Feng Z and Shi Y: Extracorporeal membrane oxygenation (ECMO): Does it have a role in the treatment of severe COVID-19? Int J Infect Dis 94: 78-80, 2020.

76. Ramanathan K, Antognini D, Combes A, Paden M, Zakhary B, Ogino M, MacLaren G, Brodie D and Shekar K: Planning and provision of ECMO services for severe ARDS during the COVID-19 pandemic and other outbreaks of emerging infectious diseases. Lancet Respir Med 8: 518-526, 2020.

77. Cho HJ, Heinsar S, Jeong IS, Shekar K, Li Bassi G, Jung JS, Suen JY and Fraser JF: ECMO use in COVID-19: Lessons from past respiratory virus outbreaks-a narrative review. Crit Care 24: 301, 2020.

78. Atluri S, Manchikanti L and Hirsch JA: Expanded Umbilical Cord Mesenchymal Stem Cells (UC-MSCs) as a Therapeutic Strategy in Managing Critically Ill COVID-19 Patients: The Case for Compassionate Use. Pain Physician 23: E71-E83, 2020.

79. Tovar I, Guerrero R, López-Peñalver JJ, Expósito J and Ruiz de Almodóvar JM: Rationale for the Use of Radiation-Activated Mesenchymal Stromal/Stem Cells in Acute Respiratory Distress Syndrome. Cells 9: E2015, 2020.

80. Kavianpour M, Saleh M and Verdi J: The role of mesenchymal stromal cells in immune modulation of COVID-19: Focus on cytokine storm. Stem Cell Res Ther 11: 404, 2020. 